\title{
LA TEORÍA BUDDHISTA DE LOS DHARMAS
}

\author{
María Teresa ROMÁN \\ UNED
}

Abraham VÉLEZ

\section{Introducción}

Desde una perspectiva histórica, el pensamiento buddhista de la India suele dividirse en tres grandes fases:

1) Buddhismo antiguo o pre-escolástico, desde la época del Buddha (ca. 560-480 a.C.) hasta la escisión del buddhismo en escuelas (ca. 350 a.C.). En este perfodo se recopilan los sermones (Sütras)del Buddha y las reglas disciplinarias (Vinaya) de la congregación (sangha) de discípulos.

2) Buddhismo escolástico o abhidhármico, desde alrededor del 350 a.C. hasta el siglo I. a.C. ${ }^{1}$ En este período se ordenan y sistematizan las ensenanzas del Buddha. Se analizan los fenómenos mentales y sus relaciones condicionales. Aparecen diversas interpretaciones de la Enseñanza (Dharma) y el buddhismo se diversifica en escuelas. Cada escuela elabora sus propios textos sistemáticos (Abhidharma). Se habla tradicionalmente de 18 escuelas pero conocemos el nombre de más de treinta. Los únicos abhidharmas que se han conservado en su integridad son los de las escuelas Theravada (en lengua pâli) y la Sarvâstivada (en traducción china). La escuela Theravâda es la única de esta época que sigue existiendo, fundamentalmente en Sri Lanka, Tailandia y Birmania.

1 En rigor, el buddhismo escolástico no desapareció en el siglo I a.C., sino que, por una parte, coexistió con el buddhismo posterior y, por otra, fue integrado en el buddhismo Mahâyâna. 
3) Buddhismo Mahâyâna, desde el siglo I. a.C. hasta la desaparición del

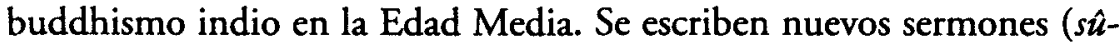
tras) que son atribuidos al Buddha histórico. Las escuelas filosóficas principales son la Mâdhyamaka y la Yogácâra. El buddhismo cruza las fronteras indias y se extiende por Tibet, China, Korea, Japón, etc.

Aunque hay grandes diferencias teóricas y prácticas entre las distintas escuelas y épocas del buddhismo, la filosofía buddhista presenta una sorprendente continuidad doctrinal. Por supuesto, como en todas las tradiciones, hay discontinuidades y tesis heterodoxas que asemejan el buddhismo al hinduismo o al materialismo, sin embargo, predomina la unidad y continuidad de la tradición (dentro y fuera de la India) en cuanto a las tesis filosóficas fundamentales se refiere ${ }^{2}$.

Una de las doctrinas filosóficas que ejemplifica el devenir filosófico de la tradición buddhista (predominio de la unidad en la diversidad y existencia de algunas desviaciones heterodoxas), es la doctrina de los dharmas o componentes de la existencia.

Como señala E. Conze, "la teoria de los dharmas es común a todas las escueLas y proporciona el marco dentro del cual opera la sabiduría buddhista"3.

La teoría de los dharmas no sólo es común a todas las escuelas buddhistas sino que para muchos especialistas, es una de las doctrinas más importantes del buddhismo. Así por ejemplo, $\mathrm{H}$. Von Glassenapp afirma que era "el núcleo de la dogmática Buddhista ya en la época de Ashoka (alrededor del 250 a.C.)"4. Igualmente, T. Stcherbatsky afirma que "la concepcion del dharma es el punto central de la doctrina buddhista" 5 y T. R. V. Murti afirma que la teoría de los dharmas, es una concepción central para el sistema buddhista Vaibhasika "así como lo es para otros sistemas de buddhismo"6.

La teoría de los dharmas es también una de las grandes aportaciones del buddhismo a la filosofía. Si bien algunos autores como T. Stcherbatsky defien-

2 Véase por ejemplo la obra de D. Kalupahana, "A History of Buddhist Philosophy”, University of Hawaii Press, Honolulu, 1992.

3 E. Conze, "Buddhist Thought in Indid", Allen \& Unwin: Londres, 1962, p. 92.

4 H. von GlasenAPP, "La filosofla de los hindúes", Barral, Barcelona, 1977, p. 318.

5 T. STCHERBATSKY, "Central Conception of Buddhism", Londres, 1924; hay traducción castellana: "Dharma el concepto central del budismo", Sirio, Málaga, 1994, p. 119.

6 T. R. V. MurTI, "The Central Philosophy of Buddhism", Allen \& Unwin, Londres, 1955, p. 69. 
den que la teoría de los dharmas es fruto de la influencia de la filosofia Sânkhya sobre el buddhismo ${ }^{7}$, lo cierto es que la teorla de los dharmas es una innovación buddhista. Como afirma H. Von Glassenapp la filosofía Sânkhya, "surgió varios siglos después de Buddha... Más bien se puede pensar que el no-tetsmo y otras teorias sostenidas por el Sânkhya fueron formuladas por primera vez bajo la influencia buddhista" 8 .

T. Stcherbatsky afirma también que la teoría de los dharmas buddhista deriva de la Kâthaka Upanishad hinduista9. Sin embargo, está demostrado que dicha Upanishad no sólo es posterior al surgimiento del buddhismo sino que está profundamente influida por él. Como señala $\mathrm{H}$. Nakamura, "no podemos cerrar los ojos por más tiempo al hecho de que a través de toda la Kâthaka Upanishad, las huellas del buddhismo antiguo son notables" 10.

La teoría de los dharmas, como la práctica totalidad de la filosofía india y extremo oriental, ha pasado prácticamentẹ desapercibida para el pensamiento europeo y occidental. Para subsanar este desconocimiento, sería necesario superar el etnocentrismo filosófico occidental e investigar desde una perspectiva histórico-crítica las filosofías de otras culturas. En el mundo actual, sería conveniente globalizar también la filosofía y enriquecerla con las tradiciones filosóficas no occidentales ${ }^{11}$.

Que la filosofía occidental debería abrirse y salir de su aislamiento, es algo que no requiere justificación, basta con reflexionar sobre el hecho de que hasta la física cuántica del siglo $\mathrm{xx}$, la filosofía de los procesos de Whitehead, la teoría general de sistemas de Von Bertalanffy, el correlacionalismo de Amor Ruibal y la ontología de Zubiri, el pensamiento occidental no había elaborado una concepción de la realidad no substancialista, constitutivamente relacional (respectiva) e interdependiente, es decir, una concepción de la realidad semejante a la que se desprende de la teoría de los dharmas buddhista formulada entre los siglos v-III a.C.

7 Véase por ejemplo T. STCHERBATSKY, op. cit., p. 53.

8 H. vON GlasenaPP, op. cit., p. 319.

9 T. STCHERBATSKY, op. cit., p. 113.

10 H. Nakamura, "A History of Early Vedânta Philosophy", MB, Nueva Delhi, 1983, p. 27.

11 Sobre la problemática de si hay o no filosofía en la India, puede verse la obra de Raimón PANIKKaR “La experiencia filosófica de la India”, Trotta, Madrid, 1997. 
En este artículo explicaremos brevemente la historia filosófica de la teoría de los dharmas desde el Buddha hasta Nâgârjuna ${ }^{12}$, es decir, en el buddhismo preescolástico, en el abhidhármico o escolástico, y en la escuela Mâdhyamaka del buddhismo Mahâyana ${ }^{13}$.

El artículo se divide en tres partes: en la primera explicamos (a partir de los sermones más antiguos del buddhismo) ${ }^{14}$ la concepción que tiene el Buddha histórico sobre los dharmas. En la segunda, explicaremos las concepciones de las escuelas Theravada y Sarvâstivâda del buddhismo abhidhármico. En la tercera, explicaremos la concepción Madhyamaka de los dharmas a partir de la obra fundamental de Nâgârjuna, los Mûlamadhyamakakarikâ o Versos sobre los fundamentos del camino medio.

\section{Los dharmas en el buddhismo pre-escolástico}

La finalidad de la filosofía buddhista en general y la doctrina de los dharmas en particular, es soteriológica, es decir, pretende conducir al hombre desde un estado de sufrimiento hasta un estado de felicidad o extinción del sufrimiento denominado Niruâna (Nibbana en pâli).

Para llegar a dicho fin, es necesario transformar y sustituir ciertos actitudes, pautas de comportamiento y formas de percibir las cosas que producen sufrimiento, por otras actitudes, pautas de comportamiento y formas de percibir las cosas que generan felicidad. Dado que para el buddhismo el estado de nuestra mente es la raíz última del sufrimiento y la felicidad, el objetivo básico, es sustituir ciertos estados mentales como el odio, el deseo ávido, la ignorancia, etc. por otros como el desapego o desprendimiento, el amor o solidaridad y la sabiduría o recta visión del mundo. "La mente es la precursora de las cosas(dhamma), su guía y artifice. Al igual que la rueda sigue la pezuña de un buey, el sufrimiento sigue al que habla o actia con una mente malevola"15.

12 Filósofo buddhista del siglo II-III d.C., fundador de la escuela Mâdhyamaka.

13 Por razones de espacio, no podemos ocuparnos de la escuela Yogâcâra.

14 Nos referimos a las cuatro primeras colecciones de sermones (Sutta Pitaka) del canon pâli (Anguttara Nikâya, Dîgha Nikâya, Majjbima Nikâya y Samyutta Nikâya) y a sus equivalentes en lengua china (âgamas), y algunos fragmentos de la quinta colección de sermones como por ejemplo el Sutta Nipáta.

is "Manopubbangamá dhamma manosetthà manomayà 
Para fomentar dichos actitudes, pautas de comportamiento y formas de percibir las cosas, es necesario llevar una vida ética y disciplinar la mente por medio de la atención, la concentración, el análisis racional y la contemplación meditativa de las leyes y estructura del universo.

Sólo quien llega a ver las leyes y estructura del universo (Dharma en sánscrito o Dhamma en pâli) ${ }^{16}$, puede compartir la "naturaleza" y visión del mundo que tiene un Buddha, es decir, puede alcanzar su mismo estado de despertar o iluminación. Por eso dice el Buddha que quien ve la ley y estructura del universo (Dharma), le ve a él, y quien le ve a él, ve la ley y estructura del universo (Dharma) ${ }^{17}$.

La iluminación o despertar consiste en el logro de un estado de felicidad y liberación denominado Nirvana caracterizado por la extinción del proceso psicofísico del sufrimiento, por el desapego, el amor incondicionado y la recta visión del mundo, es decir, caracterizado por ciertos factores emocionales y cognitivos ${ }^{18}$.

Uno de los factores de la iluminación es la recta visión del mundo, o lo que es lo mismo, la intuición del origen-inter-dependiente o pratîtyasamutpâda ( $p a-$ ticcasamuppada en pâli) de las cosas (dharma o dhammà en pâli).

La iluminación, la recta visión del mundo y la intuición del origen-inter-dependiente son en cierto modo equivalentes. Antes de lograr intuitivamente la recta visión del mundo, es necesario el análisis racional de la realidad y una comprensión filosófica del origen-inter-dependiente. Comprender filosóficamente el origen-inter-dependiente de las cosas, equivale a comprender la estructura constitutivamente dinámica, relacional, insubstancial e intercondicionada del universo.

Manasâ ce padutthena

bhasati va karoti vâ

Tato nam dukkhamanveti

cakkam'va vahato padam" (Dhp. 1).

16 Cuando usamos el término Dharma (Dhamma en pâli) con mayúscula, no nos referimos a los dharmas (dhammas en pâli) que son objeto de este estudio, es decir, en su acepción de "estado mental, cosa y sus componentes", sino a las acepciones de ley universal o natural, orden del universo, Enseñanza del Buddha.

17 "Yo kbo dhammam passati so mam passati, yo mam passati so dhammam passati" (S. III. 120).

18 (M. I. 167). 
El origen-inter-dependiente no sólo es uno de los contenidos de la iluminación sino que desde una perpectiva filosófica, es también el principio ontológico fundamental de la Enseñanza buddhista y la ley principal de su cosmología.

Dado que según el Buddha, su Enseñanza (Dharma) se limita a explicar el origen y cesar del sufrimiento ${ }^{19}$, comprender el origen-inter-dependiente del sufrimiento o su explicación causal, equivale a comprender la Enseñanza del Buddha: "Quien ve la Enseñanza, ve el origen-inter-dependiente y quien ve el origen-inter-dependiente ve la Enseñanza"20.

El origen-inter-dependiente implica que los estados mentales, las cosas y sus componentes, es decir lo que generalmente y en este contexto denominamos dharmas (dhammâ en pâli), son insubstanciales (anatta en pâli). No se adhieren $o$ inhieren en ninguna realidad subyacente ni son los accidentes de una substancia oculta de las cosas.

Los estados mentales, las cosas y sus componentes no existen de manera estática, permanente, eterna, subsistente, en sí o por sí mismos, independientemente $\mathrm{o}$ al margen de otros estados mentales, cosas o componentes. Al contrario, los dharmas existen respecto de otros y gracias a otros, entre y dependiendo de otros, es decir inter-dependientemente, apoyándose y soportándose mutuamente en su ser y en su obrar.

Decir que los estados mentales, las cosas y sus componentes se originan interdependientemente significa que todos, se originan y cesan a partir de causas y condiciones, junto a otros estados mentales, cosas y componentes, en relación constitutiva y por medio de otras realidades.

Para el Buddha, comprender y ver la verdadera estructura de los estados mentales, cosas y sus componentes, es decir, verlos como insubstanciales e interdependientes, es de suma importancia para la pretensión soteriológica de todo su sistema. La comprensión filosófica y la contemplación meditativa de la insubstancialidad e interdependencia de los estados mentales, las cosas y sus componentes, induce al desapego y a la intuición de la ley y estructura (Dhar$m a)$ del universo en que consiste el despertar.

19 (M. I. 140).

20 "Yo paticcasamuppadam passati so dhammam passati, yo dhammam passati so paticcasamuppadam passati". (M. I. 191) 
Creer que algún estado mental, alguna cosa o alguno de sus componentes es substancial, permanente, eterno, etc., no se ajusta a la ley del universo ni a la Enseñanza del Buddha, es fuente de ignorancia, induce al apego, a la mentalidad posesiva, al egoísmo y al odio, es decir, genera sufrimiento e impide la liberación.

Para facilitar la recta visión del mundo consistente en ver el origen-inter-dependiente y la insubstancialidad de los estados mentales, cosas y sus componentes, y por tanto, para inducir hacia el desapego y amor incondicional que la favorecen, el Buddha analiza todo (especialmente el ser humano) en sus factores constitutivos.

Tras analizar fenomenológicamente al ser humano y otras realidades, el Buddha verifica que no hay nada en ellas que cumpla los requisitos ontológicos para ser una substancia inmutable y eterna. Es decir, no hay justificación racional para considerar los estados mentales, las cosas o sus componentes como eternas y permanentes, ni motivo alguno para apegarse a ellos. Sin apego, no hay engreimiento, envidia, afán de posesión, ni razón para el egoísmo o miedo a perder lo que no es, en rigor, nuestro. Sin mentalidad arrogante, posesiva ni codiciosa, tampoco hay fundamento para disputas por la posesión de algo o el odio $^{21}$.

- La teoría de los dharmas nace a partir de los diversos tipos de análisis de la realidad humana, fundamentalmente del análisis fenomenológico de los estados mentales y físicos que lo constituyen.

La teoría de los dharmas es una consecuencia de la estructura interdependiente e insubstancial de la realidad y una forma más de justificar racionalmente la insubstancialidad e interdependencia de los estados mentales, las cosas y sus componentes. Si dichas realidades son interdependientes desde su origen, no pueden ser substanciales en el sentido de contener una esencia, substancia o naturaleza eterna e inmutable, algo que existe en sí y por sí mismo, sin la colaboración y condicionamiento de otras realidades. Si después de analizar y descomponer el ser humano en sus componentes, se descubre que no son substanciales, se deduce que son conglomerados o agregados de dharmas mutuamente condicionados e interdependientes.

21 (M. I. 111). 
La doctrina de los dharmas del buddhismo pre-escolástico, guarda relación con la finalidad soteriológica de su filosofía, su talante analítico y su concepción filosófica fundamental: el origen-inter-dependiente y la insubstancialidad.

La teoría de los dharmas se formula técnicamente y se desarrolla en la época del buddhismo escolástico o abbidhármico, por ello, no es exacto afirmar que dicha teoria existe de manera desarrollada y sistemática en la época del Buddha. Lo que sí es correcto afirmar, es que las raíces de dicha teoría se encuentran en los sermones del Buddha. Como señala Y. Karunadasa, "aunque la teoria de los dharmas es una innovacion abhidhármica, las tendencias antecedentes que llevaron a su formulación y sus ingredientes básicos pueden trazarse en las antiguas escrituras buddhistas"22.

Los sermones más antiguos del Buddha utilizan el término dharma en contextos distintos y con significados muy diferentes. Según el estudio filológico clásico de Magdalene y Wilhem Geiger, en los sermones del Buddha, el término pâli dhamma tiene más de sesenta acepciones distintas ${ }^{23}$. Parafraseando a Aristóteles se podría decir que los dharmas se dicen de muchas maneras.

La raíz etimológica del término es "dhr", que significa llevar, sostener, mantener, etc. En conformidad con dicha etimología, el peso ontológico del universo no recae sobre una substancia absoluta que está detrás del hombre o del mundo, sino que son los dharmas son las entidades que llevan, mantienen o sostienen la realidad de las cosas.

La acepción técnica posterior de la palabra dharma como "objeto último del análisis irreductible analíticamente a otras realidades", no aparece en los sermones del Buddha.

Es cierto que en los sermones hay muchas cosas que se denominan dharmas en el sentido general de realidades, y que posteriormente en la época de los abhidharmas se consideraran dharmas en el sentido de objetos últimos del análisis irreductibles analíticamente a otras realidades. Sin embargo, no toda realidad que se denomina dharma en los sermones del Buddha es última o irreductible analíticamente a otras realidades. Es decir, los dharmas del buddhismo pre-es-

22 Y. Karunadasa, "The Dhamma Theory", The Wheel Publication, n. $412 / 413$, Kandy, 1996 , p. 3.

23 M. \& G. Geiger, “Pali Dhamma”, Munich, 1921. 
colástico no siempre son los "últimos" objetos derivados del análisis ni siempre algo irreductible a otras realidades.

El buddhismo pre-escolástico analiza la realidad humana en sus componentes desde distintos puntos de vista pero sin pretender ser exhaustivo ni reducir todo a sus componentes últimos e irreductibles. El Buddha no se pronuncia sobre el numero exacto de dharmas que existen ni por su duración precisa.

Los análisis característicos del buddhismo pre-escolástico son cinco y se refieren principalmente a la realidad humana y su conocimiento: análisis en mente y materia (nâma-rûpa); en cinco agregados o khandhas; en seis elementos o dhâtus; en doce bases o ayatanas, en dieciocho elementos o dhâtus.

Los componentes que resultan de dichos análisis no son siempre últimos ni irreductibles. Como señala Y. Karunadasa, "ninguno de los cinco análisis puede considerarse exhaustivo. En cada caso, uno o más de los componentes (item) pueden volverse a analizar'24.

Así por ejemplo, el dharma o agregado denominado materia o forma material (rûpa), se puede seguir descomponiendo en otros dharmas como tierra o solidez (pathavi), agua o fluidez (apo), fuego o calor (tejo), aire (vâyu), espacio (akâsa) ${ }^{25}$. El agregado conciencia (viññana en pâli o vijñana en sánscrito), se puede subdividir en seis tipos de conciencia según al sentido (gusto, olfato, vista, tacto, oído, mente) al que corresponda. Por tanto, hay que matizar la afirmación de C. Dragonetti según la cual, los dharmas correspondientes a los cinco agregados o componentes del hombre "no pueden ser analizados ulteriormente, son irreductibles a otra $u$ otras realidades de cualquier naturaleza: el rüpa es rûpa, el vijñana es vijñana y nada más; no pueden ser descompuestos en otros elementos" 26 .

No hay que confundir los dharmas (estados mentales, cosas y sus componentes) de los sermones o Sûtrapitaka, con la teoría de los dharmas del abhidharma posterior. Como señala C. Dragonetti "la doctrina de los dharmas en el Sûtrapitaka está expuesta en forma que carece de toda sistematización'27.

24 Y. KarUnadasa, op. cit., p. 5.

25 (M. III. 239).

26 C. Dragonettr, "La doctrina de los dharmas en el Budismo", Yoga y mística de la India, Kier, Buenos Aires, 1978, p. 100.

27 C. DragonetTI, op. cit., p. 95. 
Los dharmas del buddhismo pre-escolástico no son siempre irreductibles analíticamente a otras realidades y por ello no son siempre los objetos últimos e irreductibles que componen las cosas. La filosofía de los dharmas del buddhismo pre-escolástico no es equiparable sin más a la filosofía de los dharmas del buddhismo escolástico o abhidhármico. Mientras que en los sermones, algunos dharmas no son últimos y se pueden seguir analizando o reduciendo a otras realidades que también son dharmas, en la época abhidhármica los dharmas sí son últimos e irreductibles analíticamente.

Por ello, no se puede decir que la filosofía del buddhismo pre-escolástico es pluralista en el sentido de que para el Buddha, la realidad se compone de dharmas últimos e irreductibles analíticamente. Tal interpretación pluralista y realista de la filosofía del buddhismo pre-escolástico, deriva principalmente de la obra de T. Stcherbatsky (seguido por N. Dutt, T. R. V. Murti, etc.), y comete dos graves errores: 1) desconoce que los dharmas de los que habla el Buddha no son siempre últimos ni irreductibles analíticamente; 2) proyecta injustificadamente dentro del buddhismo pre-escolástico, la concepción de los dharmas de cierto escolasticismo buddhista, concretamente, del pensamiento abhidhármico de la escuela Sarvâstivâda.

Como señala D. Kalupahana, "la concepción de dharma descrita por Stcherbatsky pertenece al periodo representado por el Abhidharma"28.

Además, la interpretación pluralista del buddhismo pre-escolástico contradice los textos del Buddha e implica una comprensión defectuosa de las implicaciones ontológicas del origen-inter-dependiente de las cosas.

El Buddha afirma que las cosas no son una y la misma realidad (sabbam ekattam) ni tampoco muchas realidades últimas e irreductibles (sabbam puthuttam). Dicho de otro modo, el buddhismo pre-escolástico se distancia tanto del monismo como del pluralismo ${ }^{29}$. Ni los dharmas son manifestaciones de una y la misma realidad absoluta, ni los dharmas son una multiplicidad de pequeñas realidades absolutas (separadas y constitutivamente no relativas).

El origen-inter-dependiente, es precisamente una posición intermedia (un camino medio según la terminología buddhista) que se distancia de los extre-

28 D. Kalupahana, "Causality: The Central Philosophy of Buddhism", The University Press of Hawaii, Honolulu, 1975, p. 70.

29 (S. II. 77). 
mos del monismo y el pluralismo. Si algo permite la explicación ontológica del origen-inter-dependiente es evitar el pluralismo filosófico y armonizar lo uno y lo múltiple sin decantarse por ninguno de los dos extremos ${ }^{30}$.

Los dharmas de los que habla el buddhismo escolástico no son substancias últimas e irreductibles que se combinan con otros dharmas o substancias últimas, según la ley del origen-inter-dependiente, entendida simplemente como la ley de la causalidad que combina a los elementos ya constituidos ontológicamente.

La interpretación pluralista del buddhismo pre-escolástico asemeja su filosofía al atomismo de Demócrito, la concepción de los elementos de Empédocles, al mecanicismo moderno, al escolasticismo Sarvâstivada, etc., pero no es consistente con el origen-inter-dependiente que defiende el Buddha, el escolasticismo Theravada y Nagârjuna.

Los textos del buddhismo pre-escolástico afirman repetidas veces que los dharmas (estados mentales, cosas y sus componentes), tienen básicamente tres características: son impermanentes (anicca), insatisfactorios (dukkba) e insubstanciales (anatta). Decir que los dharmas son insubstanciales significa que son dinámicos, transitorios y que se originan o cesan interdependientemente. Decir que los dharmas se originan y cesan inter-dependientemente no significa que primero surgen o existen, y luego una vez surgidos, se relacionan y condicionan mutuamente, sino que primeramente, para surgir o existir, necesitan siempre de la relación y el condicionamiento de otros dharmas. Es decir, la relación y el condicionamiento de los dharmas no es accidental sino constitutivo. El origeninter-dependiente no expresa únicamente la causalidad de los estados mentales, las cosas y sus componentes sino su relatividad radical y su condicionalidad constitutiva (idappaccayatâ). Por eso, la tradición ortodoxa de filosofía buddhista se opone siempre a la tesis según la cual, los dharmas tienen existencia última, propia o inherente (svabhava). La realidad de los dharmas no es nunca propia o inherente, no les pertenece ni es independiente de otras.

30 Véase por ejemplo la obra de J. MACY, "Mutual Causality in Buddhism and General Systems Theory", State University of New York Press, Nueva York, 1991. 


\section{Los dharmas en el buddhismo abhidhármico o escolástico}

La teoría abhidhármica de los dharmas es una profundización y elaboración más sistemática de los análisis de la realidad (especialmente humana) realizados por el Buddha. Como señala Y. Karunadasa, "el analisis en dhammas es una adición a los cinco modos de análisis previos. Su alcance es el mismo, el mundo de la experiencia consciente, pero sus divisiones son más precisas y exhaustivas" 31.

Al igual que en el buddhismo pre-escolástico, los análisis fenomenológicos de la época abhidhármica se realizaron con el fin de demostrar la inexistencia de una substancia inmutable y eterna en los estados mentales, las cosas y sus componentes. Dicho de otro modo, los análisis del buddhismo pre-escolástico, perseguían la misma finalidad soteriológica que los del buddhismo pre-escolástico: inducir al desapego hacia esos estados mentales, cosas y componentes: favorecer el amor incondicionado y ayudar al logro de la intuición del origen-inter-dependiente, la recta visión del mundo que acompaña a la liberación del sufrimiento.

De las múltiples acepciones de la palabra dharma en los sermones, cabe destacar seis por su relevancia filosófica:

1) Dharma como "enseñanza" o "doctrina": se refiere a la enseñanza del Buddha o a la de otras personas, es decir, a doctrinas (a veces también a las prácticas) de un maestro espiritual.

2) Dharma como "ley" u "orden natural": se refiere a las leyes naturales del universo o a la estructura y naturaleza del mundo, equivale a la ley natural.

3) harma como "característica" o "naturaleza" de algo: se refiere a la condición, cualidad o propiedad de algo como por ejemplo ser impermanente.

4) Dharma como opuesto a no-dharma: se refiere a los comportamientos y estados mentales morales, buenos, virtuosos, beneficiosos, en contraposición a los que no lo son.

5) Dharma como "cosa": sentido ontológico general y amplio, se refiere a diversos modos de ser y niveles de realidad, equivale a los términos no específicos "realidad", "ente", "algo".

31 Y. KaRUNAdaSA, op. cit., p. 6. 
6) Dharma como "estado mental": sentido psicológico y epistemológico, abarca una amplia gama de realidades psicofísicas: objetos de conocimiento, sentidos del cuerpo, mente, operaciones cognitivas, emociones, virtudes intelectuales y éticas, actitudes, fases, procesos y circunstancias que atraviesa la vida humana desde el nacimiento hasta la liberación o Nirvâna.

De las seis acepciones fundamentales de la palabra dharma en los sermones, la más importante para el surgimiento de la teoría abhidhármica de los dharmas es la última: dharma en sentido de un estado psico-epistemológico.

Los dharmas de la época abhidhármica son los "objetos últimos e irreductibles del análisis fenomenológico de la realidad humana y por extension extrahumana". Dichos dharmas no son meras creaciones de la mente pero tampoco son realidades (al menos para todas las escuelas) completamente extramentales. Ni realismo ingenuo ni idealismo absoluto es otra de las carácterísticas de la filosofia buddhista ortodoxa.

Los abhidharmas y su teoría de los dharmas, definían, clasificaban y relacionaban todo tipo de experiencias mentales o dharmas. Con ello, se trazaba un mapa minucioso de la mente humana, útil para guiarse a lo largo del camino espiritual. Gracias a las definiciones, clasificaciones y relaciones entre los distintos dharmas, el discípulo era capaz de comprender y orientarse mejor en los diferentes estados mentales por las que atravesaba, desde los inicios de la práctica espiritual hasta el logro del despertar.

Los abhidhármas y su teoría de los dharmas, ordenaban y sistematizaban todas las doctrinas buddhistas. De ese modo, no sólo era más fácil el estudio de la filosofia buddhista sino que también se podía justificar con mayor racionalidad, el origen-inter-dependiente y la insubstancialidad de los estados mentales, las cosas y sus componentes.

Sin embargo, el desarrollo y sofisticación del escolasticismo buddhista, hizo que el objetivo soteriológico original se desvirtuara. Proliferaron diversas clasificaciones e interpretaciones de los dharmas y aparecieron las primeras escuelas filosóficas divergentes del buddhismo ${ }^{32}$.

32 Es importante señalar que las primeras divisiones del buddhismo en escuelas, no obedeció tanto a diferencias doctrinales como a diferencias en el modo de vivir la disciplina monástica. Para el buddhismo, la ortopraxis siempre ha sido más importante que la ortodoxia. 
El escolasticismo buddhista convirtió los análisis fenomenológicos de las experiencias humanas y su finalidad soteriológica, en teorías filosóficas acerca del tipo de existencia, duración y número exacto de los dharmas. Lo que en su origen era un medio para la superación de las concepciones especulativas sobre la realidad (fundamentalmente las que defendían una substancia inmutable y eterna en el ser humano), se transformó en un conjunto de concepciones especulativas sobre los dharmas. Se comenzó a discrepar sobre la duración y número exacto de dharmas que componían la mente humana, y se elaboraron teorías sobre la realidad de los dharmas. sólo mental, en parte mental y en parte extramental, completamente extramental, presente, futura o pasada, etc. Cada escuela desarrolló sus propios textos sistemáticos o Abhidharma, y los añadió a los textos canónicos de los sermones (Sütras) y la disciplina monástica (Vinaya), afirmando que fueron compuestos por el propio Buddha.

Del escolasticismo abhidhármico y sus especulaciones sobre la naturaleza sólo mental, mental y extramental de los dharmas, etc., surgieron las filosofias buddhistas de carácter realista e idealista y sus tendencias pluralistas y monistas respectivamente.

De todas las escuelas de esta época, sólo se han conservado los abhidharmas o textos sistemáticos de las escuela Sarvastivada y Theravada.

Ambas escuelas desarrollaron su propia teoría de los dharmas pero con presupuestos filosóficos distintos. Mientras la teoría Theravada de los dharmas, si bien con tendencia al realismo, permanecía fiel a la filosofía no substancialista, no monista ni pluralista del buddhismo pre-escolástico, la Sarvastivâda desarrolló una concepción de los dharmas ultrarealista, substancialista y pluralista.

La filosofía Theravada no admitía la tesis de los puggalavadines según la cual, en sentido último y real, existe una entidad denominada individuo (puggala) aparte de sus componentes. En contraposición a dicha doctrina, la escuela Theravada defiende que en sentido último y real, existen únicamente los agregados o dhammas que constituyen al ser humano ${ }^{33}$. El concepto de individuo, no tiene referencia objetiva ya que sólo se refiere al conjunto de los cinco agregados o dhammas que conforman la realidad humana. El individuo existe convencionalmente pero no en sentido último ni real, ya que los agregados y los dhammas que lo constituyen son lo único propiamente último y real. Aho-

33 (Kvu. I. 1ss). 
ra bien, los dhammas que existen en sentido último (paramattha) y real (saccikattha) para la escuela Theravada, no existen de manera substancial (en y por sí mismos, inherentemente) ni independientemente (sin relación constitutiva) de otros.

Los dhammas de los que habla el Abhidhamma y la tradición comentarial de la escuela Theravâda, son en todos los casos insubstanciales y están siempre sometidos al origen-inter-dependiente. La única excepción a este respecto es el Nibbâna (Nirvana en sánscrito) que es insubstancial pero no sometido al origen-inter-dependiente ${ }^{34}$.

Cada dhamma es último porque no es reductible analíticamente a otros dhammas y es real porque a diferencia de otros conceptos como el de individuo, sí tiene un correlato objetivo, sí existe en realidad, si bien de manera insubstancial e interdependiente.

Uno de los primeros textos escolásticos que no se incluyen en el Abhidhamma Pitaka del Theravada sino en la quinta colección del Sutta Pitaka, es el Patisambbidâmagga. Alli se dice explícitamente que los agregados están vacíos o carecen de existencia substancial, inherente o propia (sabhavena-suññam). Por ello, los dhammas en que el Abhidhamma descompone los agregados, no son interpretados como poseyendo existencia substancial, propia o inherente. Cuando el Abhidhamma Theravâda utiliza el término sabhava (svabhavva en sánscrito), no lo hace en el sentido de existencia substancial, propia o inherente que tiene para la escuela Sarvâstivâda, sino en el sentido de "característica fenomenológica principal o naturaleza propia de algo". Cuando la tradición Theravâda posterior al Abhidhamma usa el término sabhâva como sinónimo de dhamma, no quiere decir que los dhammas tienen existencia inherente o propia sino únicamente cierta característica principal o naturaleza propia que lo distingue y hace irreductible a otros dhammas. Como afirma Y. Karunadasa "la definicion de dhamma como aquello que conlleva (bears) su propia naturaleza significa que cualquier dhamma representa un hecho distinto de la existencia empirica, el cual no es compartido por otros dhammas. Por ello, sabhâva es también definido como aquello que no es poseido en común por otros (anaññasadhârana), como la

34 Aunque en rigor el Nibbana transciende tanto la insubstancialidad como el origen-inter-dependiente, y por tanto, no es necesario afirmar que el Nibbâna tampoco es una substancia (atman), las interpretaciones atmanistas del Nibbâna en este siglo, han hecho que la hermenéutica Theravâda contemporánea defienda la insubstancialidad del Nibbâna pero no su origen-inter-dependiente. 
naturaleza peculiar a cada dharma (âvenika-sabhâva), y en tanto que naturaleza propia no es predicable de otros dhammas (asaddhârana-sabhâva)"35.

Los dhammas son distinguibles conceptualmente pero no separables realmente, son respectivos, mutuamente condicionados y constitutivamente interdependientes. Forman una unidad que no elimina la diversidad, no se reducen a una sóla realidad (monismo) pero tampoco son un conjunto de realidades separadas (pluralismo) que una vez constituidas, se relacionan entre si según la ley de causalidad.

La concepción Sarvastivada de los dharmas difiere considerablemente de la Theravada, de hecho, el Abhidhamma Theravada rechaza explícitamente la filosofia Sarvastivada ${ }^{36}$. Para la escuela Sarvastivada, los dharmas están separados realmente, tanto espacial como temporalmente, porque cada uno de ellos existe instantánea o momentáneamente (kshanika).

Para justificar la continuidad de las cosas a través de la sucesión discontinua de dharmas instantáneos, la escuelas Sarvâstivâda afirma que los dharmas tienen una esencia ideal (svabhâva) y múltiples manifestaciones momentáneas.

La esencia ideal o sutil de cada dharma está al margen del origen-inter-dependiente, es inmutable y existe siempre, en el pasado, en el presente y en el futuro. De aquí deriva el nombre de la escuela: todo existe (Sarvam asti). Para el Sarvâstivada, los dharmas tienen svabhâva pero no únicamente en el sentido de tener naturaleza o características propias como afirma la escuela Theravada, sino también en el sentido de ser algo distinto de las manifestaciones instantáneas de los dharmas; y algo, que (a diferencia de las manifestaciones instantáneas de los dharmas), existe siempre de manera propia, inherente, substancial, es decir, al margen de las múltiples manifestaciones de los dharmas. .

La instantaneidad o momentariedad (kshanika) de cada dharma y la distinción ontológica entre esencia ideal (svabhâva) y manifestaciones instantáneas, implican una concepción pluralista y ultrarealista de los dharmas. Lo verdaderamente real, la esencia ideal de un dharma está separada realmente de las esencias ideales de los otros dharmas. Las manifestaciones instantáneas de los dharmas también están separadas tanto espacial como temporalmente porque existen durante un momento. Como señala T. Scherbatsky atribuyendo erró-

36 (Kvu. I. 5ss). 
neamente dicha concepción de los dharmas a todo el buddhismo antiguo, "un elemento buddhista es siempre una entidad separada y no es ni un 'compuesto' ni un 'fenómeno' sino sólo un elemento (dharma)" 37.

Los dharmas no se mueven sino que desaparecen cuando acaba un momento y vuelven a aparecer en el momento siguiente. Los dharmas pasan continuamente del ser al no ser, a cada instante y sin solución de continuidad. Para que el tránsito del ser al no ser sea posible, los sarvâstivadines tienen que presuponer una esencia ideal o dharma sutil transcendente que garantiza la identidad y continuidad de todos los procesos o manifestaciones momentáneas de esos dharmas. El universo Sarvâstivâda es como un entramado de flashes intermitentes, luces discontinuas que se encienden y se apagan sin cesar. En palabras de T. Scherbatsky, "los elementos de la existencia son apariencias momentáneas, relámpagos en el mundo de los fenómenos que provienen de una fuente desconocida. Al igual que están desconectados, por decirlo de algún modo, en extension, no estando encadenados por ninguna substancia que lo penetre todo, asi también están desconectados en profundidad y en duración, ya que duran solamente un instante (ksana). Desapareciendo tan pronto como aparecen, siendo seguidos un instante después por otra existencia igualmente fugaz"38.

Aunque la escuela Sarvâstivâda trató de permanecer fiel a la insubstancialidad del ser humano predicada por el Buddha, lo cierto es que al predicar la existencia inherente de los dharmas, defiende una forma sutil y sofisticada de substancialidad. La substancialidad de los dharmas, su existencia inherente (svabhâva), entra en contradicción con el origen-inter-dependiente. La momentariedad de los dharmas implica una visión pluralista de los dharmas, y por tanto, el camino medio entre la unidad y la pluralidad del buddhismo pre-escolástico. Las realidades respectivas y constitutivamente condicionadas que eran los dharmas en el Budddhismo pre-escolástico y la escuela Theravâda, pasan a ser una especie de átomos separados e independientes que componen las cosas. De un modelo orgánico del universo y de una multicausalidad constitutiva de las cosas, se pasa a un modelo mecanicista y a una causalidad meramente lineal ${ }^{39}$.

La existencia inherente o svabhava de los dharmas durante el pasado, presente y futuro, conculca el origen-inter-dependiente ya que se afirma la exis-

37 T. STCHERBATSKY, op. cit., p. 63.

38 T. STCHERBATSKY, op. cit., p. 73.

39 J. MaCY, op. cit., pp. 7-19. 
tencia de cosas que no se originan ni cesan. Además el origen-inter-dependiente se desvirtúa y se convierte simplemente en causalidad y no también en la funcionalidad o condicionalidad constitutiva y radical de lo real. Los dharmas no son en función de otros como se desprende del origen-inter-dependiente sino que existiendo inherentemente desde siempre, se combinan causalmente.

\section{La teoría Madhyamaka de los dharmas}

Para devolver al Abhidharma su espiritu original y restablecer la filosofía de los sermones del Buddha, aparecieron los sermones sobre la perfección de la sabiduría

(Prajñâparamitâ Sûtras) y el pensamiento de Nâgârjuna, fundador de la escuela Mâdhyamaka en el siglo II d.C. Su obra principal es "Versos sobre los fundamentos del camino medio (Mûlamadhyamakakarikâ). En dicha obra, se lleva a cabo una pormenorizada refutación del substancialismo escolástico de la escuela Sarvastivada. Como señala G. Tucci refiriéndose a Nâgârjuna, "lo que él trata de establecer con coherencia logica es la verdad del Vacio... y por lo tanto, lo que el está obligado a refutar es la concepción dogmática y realista de los Sarvâstivadins" 40 .

En el mismo sentido, D. Kalupahana afirma que Nâgârjuna "intenta desacreditar las opiniones heterodoxas, especialmente aquellas de los Sarvâstivadines $y$ Sautrantikas" 41 . Igualmente N. Dutt, afirma que Nâgârjuna "toma en consideración las opiniones hinayânistas, principalmente la de los Sarvâstivadines y muestra los puntos débiles de sus opiniones"42.

Nâgârjuna pretende combatir las desviaciones substancialistas de cierto escolasticismo buddhista y no de todo buddhismo anterior a él, no para revolucionar la filosofia buddhista e iniciar una nueva escuela de pensamiento, sino para restablecer la filosofía ortodoxa de la tradición ${ }^{43}$. Para refutar el substan-

40 G. Tucci, "La escuela idealista de Buddhismo", Revista de Estudios Budistas, n. 11, México y Buenos Aires, 1996, p. 50

41 D. Kallinhana, Mûlamadhyamakakarika of Nagârjuna, State University of Nueva York, Nueva York, 198t, p. 26.

42 N. DutT, Mahàyana Buddhism, Nueva Delhi, 1978, p. 226.

43 A. Velez, "Nâgârjuna: ¿revolución o continuación de la filosoffa del Buddha?", Actas del II Encuentro Español de Indologia, Universidad de Salamanca, 1997. 
cialismo de la escuela Sarvâstivâda o su concepción de existencia inherente en los dharmas, Nâgârjuna utiliza el método de reducción al absurdo: si los dharmas tienen existencia inherente o propia, se siguen consecuencias irracionales o contraproducentes para la práctica espiritual. Ya en el primer capítulo de su obra afirma lo siguiente: "Si las cosas no existieran sin existencia propia, la frase: 'cuando esto existe, eso es', no seria posible"44. Este verso hace referencia a la formulación abstracta clásica del origen-inter-dependiente: "Cuando esto es, eso existe, al originarse esto, eso se origina. Cuando esto no es, eso no existe, con cesar de esto, eso cesa"45.

La formulación abstracta clásica del origen-inter-dependiente equivale a la totalidad de la Enseñanza del Buddha y a la ley fundamental de la cosmología. Al aludir a dicha formulación, Nâgârjuna está defendiendo la filosofía tradicional buddhista y planteando un dilema entre la existencia inherente (svabhâva) y el origen-inter-dependiente: si hay existencia inherente, se conculca el origeninter-dependiente y el buddhismo no es posible. Lo que existe inherentemente no puede ser destruido ni transformarse desde el sufrimiento hasta la felicidad, si el sufrimiento o cualquiera de los dharmas que condicionan el sufrimiento existe inherentemente, el logro de su cesar no es posible.

Por tanto, al igual que para Buddha el mundo está vacío de existencia substancial, para Nâgârjuna el mundo está vacío de existencia inherente o propia. De otro modo, no sería posible la liberación del sufrimiento. La ausencia de existencia inherente en los dharmas, implica que se originan y cesan inter-dependientemente, es decir que están vacíos de existencia substancial. Todo lo sometido al origen-inter-dependiente y a la vacuidad de existencia substancial, es una designación convencional mediada lingüísticamente, es decir, un camino medio ontológico (ni ser ni no ser, ni uno ni muchos), epistemológico (ni realismo ni idealismo) y lingüístico (ni existencia ultrarealista de conceptos, $\mathrm{ni}$ mera existencia mental de los mismos): "Lo que surge-inter-dependientemente, es denominado vacuidad. Eso es una designación convencional y es el camino medio"46.

44 "Bhâvanàm nihsvabhavânâm na sattâ vidyate yatah, satîdam asmin bhavatîty etan naivopapadyate"(M.M.K. I. 10).

45 "Imasmim sati idam hoti, imass, uppada idam uppajjati. Imasmim asati idam na hoti, imassa nirodha idam nirujjati", (S. II. 28), etc.

46 "Yah pratîtyasamutpâdah sûnyatàm tâm pracaksmabe sâ prajñaptir upâdâya pratipat saiva madhyamâ" (MMK.XXIV. 18). 
El camino medio, estar vacío de existencia inherente o propia, o lo que es lo mismo, originarse y cesar inter-dependientemente, no significa inexistencia en absoluto (no ser) ni existencia absoluta (ser). La realidad de los dharmas consiste en originarse y cesar de manera insubstancial (vacuidad) y según el principio del origen-inter-dependiente, es decir, de un modo que no puede calificarse adecuadamente ni de ser ni de no ser. La realidad deviene y el devenir no es propiamente ser pero tampoco puro no ser. Es vacuidad y origeninter-dependiente, el camino medio del Buddha para vencer el dilema entre lo que es y no es. Como reconoce Nâgârjuna, "en el discurso a Kâtyâyana, las teorías del ser y del no ser han sido superadas por el Bienaventurado ${ }^{47}$, sabedor de lo real e irreal" 48 .

Para el Mâdhyamaka, la vacuidad de las cosas no significa tampoco que hay algo absoluto llamado vacuidad que subyace a todas las cosas. La vacuidad significa que las cosas y los dharmas que las constituyen están vacías de un tipo de existencia determinada, la existencia propia o inherente. La vacuidad no es un dharma entre otros o una entidad positiva, sino la privación de un tipo de existencia en todos los dharmas incluido el Nirvâna que aparece en las clasificaciones de la época abhidhármica. La famosa afirmación de Nâgârjuna según la cual no hay diferencia entre los dharmas condicionados y el dharma incondicionado (Nirvâna), no significa que sean lo mismo (monismo), que tengan el mismo peso ontológico, que de igual una cosa que la otra o que no es necesario transformar el sufrimiento en felicidad (lo que haría la práctica del buddhismo inconsistente e innecesaria, justo lo contrario de lo que Nâgârjuna pretende en su obra). Más bien, la no diferencia (no se dice identidad ya que implicaría monismo) entre el sufrimiento y la felicidad, significa por una parte, que ambos son insubstanciales o vacuidad, y por otra, que están interrelacionados o no separados de algún modo (de lo contrario se incurriría en pluralismo. El Nirvâna del que se habla y especula en todas las clasificaciones de dharmas características de la época abhidhármica como el dharma incondicionado, es al igual que todos los demás conceptos sobre el sufrimiento, una designación convencional mediada lingüísticamente y por tanto, sometida al origen-inter-depndiente e insubstancial, vacía de existencia inherente o propia.

47 Término honorífico para el Buddha.

48 "Kàtyâyanavade câstî ti nâstî ti cobhayam pratisiddham bhagavatha bhâvabhâva-vibhâvina” (M.M.K.XV. 7). 


\section{Bibliografía}

\section{Fuentes}

Sermones del Buddha (Sutta Pitaka) del canon pâli (edición de la Pâli Text Society): Anguttara Nikaya. (A)

Dîgha Nikâya. (D)

Majjhima Nikâya. (M)

Samyutta Nikàya. (S)

Sutta Nipàta. (Sn)

Dhammapada (Dhp)

Patisambhidâmagga (Psm)

Abhidhamma Pitaka del canon pâli (edición de la Pâli Text Society):

Dhammasanganî (Dhs)

Kathâvatthu (Kvu).

Obras de Nâgârjuna:

$M$ lamadhyamakakârika, $M$ lamadhyamakakarika of Nagarjuna, Nueva York: State University of Nueva York (edición sánscrita y traducción inglesa de D. Kalupahana), 1986.

Obras de Vasubandhu:

Abhidharmakosa, L'Abhidharmakosa de Vasubandhu, Bruxelles: Institut Belge des Hautes Études Chinoises (traducción francesa de L. De la Vallee Poussin, 1980.

\section{Estudios}

ConZe, E., Buddhist Thought in India, Londres: Allen \& Unwin, 1962.

Dragonetti, C., La doctrina de los dharmas en el Budismo, Yoga y mistica de la India, Buenos Aires: Kier, 1978.

- Udana, la palabra de Buda, Barcelona: Barral, 1971.

Dutr, N., Mahâyâna Buddhism, Nueva Delhi: MB, 1978.

FAtone, V., El budismo "nibilista", Buenos Aires: Editorial Universitaria de Buenos Aires, 1962. 
Geiger, M. \& G. Pali, Dhamma, Munich, 1921.

Kalupahana, D., Causality: The Central Philosophy of Buddhism, Honolulu: The University Press of Hawaii, 1975.

- A History of Buddhist Philosophy, Honolulu: University of Hawaii Press, 1992.

MACY, J., Mutual Causality in Buddhism and General Systems Theory, Nueva York: State University of New York Press, 1991.

MurTI, T. R. V., The Central Philosophy of Buddhism, Londres: Allen \& Unwin, 1955.

Nakamura, H., A History of Early Vedanta Philosophy, Nueva Delhi: MB, 1983.

PANikKar, R., La experiencia filosofica de la India, Madrid: Trotta, 1997.

PARAIN, B. (dir.): «El pensamiento prefilosófico y oriental”, en Historia de la Filosofia, Vol. I., Madrid: Siglo XXI, 1984.

Román, M. T., Buda: Sendero del alma, Madrid: Aula abierta (UNED), 1997.

STCHERBATsKy, T., Central Conception of Buddhism, Londres 1924 (traducción castellana: Dharma el concepto central del budismo, Málaga: Sirio, 1994).

Vélez, A., Buddha, Madrid: Ediciones del Orto, 1998 (en prensa).

VON GLASENAPP, H., La filosofia de los hindúes, Barcelona: Barral, 1977.

Zimmer, H., Filosofias de la India, Buenos Aires: Editorial Universitaria de Buenos Aires, 1979.

\section{Artículos}

DragonetTI, C., «La doctrina de los dharmas en el Budismo", Boletín de la Asociación Española de Orientalistas, Madrid, 1977.

Karunadasa, Y., "The Dhamma Theory», The Wheel Publication, n. ${ }^{\circ} 412 / 413$, Kandy 1996.

RomÁn, M. T., "Buda: un personaje para la historia del antiguo oriente», Espacio, Tiempo y Forma, UNED, Madrid, 1994.

TuccI, G., "La escuela idealista de Buddhismo", Revista de Estudios Budistas, n. ${ }^{\circ} 11$, México y Buenos Aires, 1996.

VÉLEZ, A., "Nâgârjuna: revolución o continuación de la filosofía del Buddha?", Actas del II Encuentro español de Indologia, Universidad de Salamanca, 1997.

- "Budismo: algunos malentendidos», Religión y Cultura, 1995. 